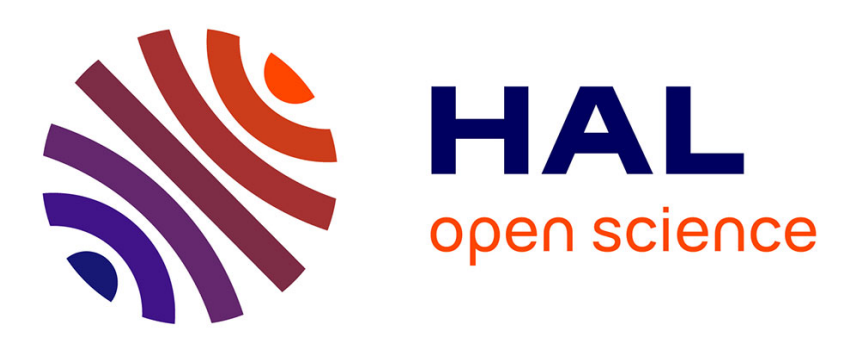

\title{
Semi-Blind Deconvolution for Resolution Enhancement in Ultrasound Imaging
}

\author{
Renaud Morin, Stéphanie Bidon, Adrian Basarab, Denis Kouamé
}

\section{To cite this version:}

Renaud Morin, Stéphanie Bidon, Adrian Basarab, Denis Kouamé. Semi-Blind Deconvolution for Resolution Enhancement in Ultrasound Imaging. IEEE International Conference on Image Processing - ICIP 2013, Sep 2013, Melbourne, Australia. pp. 1413-1417. hal-01148283

\section{HAL Id: hal-01148283 https://hal.science/hal-01148283}

Submitted on 4 May 2015

HAL is a multi-disciplinary open access archive for the deposit and dissemination of scientific research documents, whether they are published or not. The documents may come from teaching and research institutions in France or abroad, or from public or private research centers.
L'archive ouverte pluridisciplinaire HAL, est destinée au dépôt et à la diffusion de documents scientifiques de niveau recherche, publiés ou non, émanant des établissements d'enseignement et de recherche français ou étrangers, des laboratoires publics ou privés. 


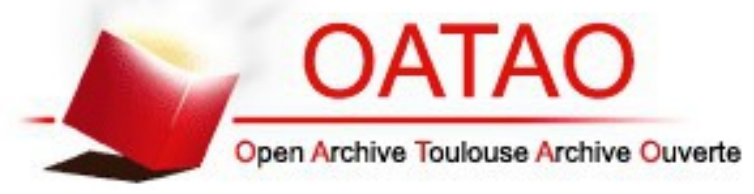

\section{Open Archive TOULOUSE Archive Ouverte (OATAO)}

OATAO is an open access repository that collects the work of Toulouse researchers and makes it freely available over the web where possible.

This is an author-deposited version published in : http://oatao.univ-toulouse.fr/ Eprints ID : 12460

To link to this article : DOI :10.1109/ICIP.2013.6738290

URL : http://dx.doi.org/10.1109/ICIP.2013.6738290

To cite this version : Morin, Renaud and Bidon, Stéphanie and Basarab, Adrian and Kouamé, Denis Semi-Blind Deconvolution for Resolution Enhancement in Ultrasound Imaging. (2013) In: IEEE International Conference on Image Processing - ICIP 2013, 15 September 2013 - 18 September 2013 (Melbourne, Australia).

Any correspondance concerning this service should be sent to the repository administrator: staff-oatao@,listes-diff.inp-toulouse.fr 


\title{
SEMI-BLIND DECONVOLUTION FOR RESOLUTION ENHANCEMENT IN ULTRASOUND IMAGING
}

\author{
Renaud Morin $^{* \dagger}$, Stéphanie Bidon ${ }^{\ddagger}$, Adrian Basarab ${ }^{\dagger}$, Denis Kouamé $^{\dagger}$ \\ $\dagger$ University of Toulouse, IRIT, UMR CNRS 5505, Toulouse, France \\ $\ddagger$ University of Toulouse, ISAE, Dept. of Electronics, Optronics and Signal, Toulouse, France
}

\begin{abstract}
In the field of ultrasound imaging, resolution enhancement is an up-to-date challenging task. Many device-based approaches have been proposed to overcome the low resolution nature of ultrasound images but very few works deal with post-processing methods. This paper investigates a novel approach based on semi-blind deconvolution formulation and alternating direction method framework in order to perform the ultrasound image restoration task. The algorithm performance is addressed using optical images and synthetic ultrasound data for a various range of criteria. The results demonstrate that our technique is more robust to uncertainties in the a priori ultrasonic pulse than classical non-blind deconvolution methods.
\end{abstract}

Index Terms - Resolution enhancement, deconvolution, ultrasound, alternating direction method of multipliers.

\section{INTRODUCTION}

Compared with other medical imaging modalities such as, e.g., X-ray computed tomography (CT), ultrasound (US) imaging is a non-invasive, cost-effective, and harmless modality commonly used in the detection of various pathologies and in the assessment of blood flow velocity. US imaging has now become a standard procedure for medical diagnosis such as breast cancer early detection $[1,2]$. However, compared with other medical imaging modalities such as magnetic resonance imaging (MRI), the resolution of US images depends on the working frequency and is very low. Additionally, the resolution is degraded due to the presence of an intrinsic noise (i.e., the speckle), the geometry of ultrasound transducers, and the system impulse response or point spread function (PSF).

US image resolution improvement is generally achieved by optimizing the imaging device, e.g., [3, 4]. Because these techniques are often highly device- and frequencydependent, an alternative to perform this task is to consider post-processing resolution enhancement techniques. A few works have investigated such techniques in US imaging and the PSF estimation, a key step in the deconvolution approaches, is still an ongoing challenge.

*Part of this work has been supported by a Région Midi-Pyrénées grant.
Lately, deconvolution techniques have been extensively studied in the general image domain to perform the estimation of a high resolution (HR) image. Early attempts to perform this task include total variation (TV) deconvolution [5] and complex deconvolution framework [6], but more recent works have focused on multiple frame super-resolution (SR) [7, 8], image restoration based on tetrolets shrinkage [9], deconvolution with an inaccurate optical PSF [10], parametric inverse filtering [11, 12], and blind deconvolution without SR [13]. Among the previously mentioned methods, blind deconvolution is a category of efficient but challenging approaches since both the HR image and the unknown blur must be estimated from the observed image. Depending on the step where the PSF is computed, these techniques can be classified into disjoint or joint estimation methods [14, 15]. In the former class, the PSF is computed separately from the original image and is then used as a priori information into an image restoration task. The latter provides the simultaneous estimation of the HR image and blur and requires a regularization process using prior knowledge about the image formation model.

In this paper, we focus on the joint estimation of the US HR image and blur with partial information about the PSF, hence the term "semi-blind" deconvolution. The classical image formation model leads to an ill-posed inverse problem solved as a semi-blind deconvolution problem for resolution enhancement. In [16], we have proposed an approach to solve this resolution enhancement problem in which the PSF was known. Here, we investigate the realistic unknown PSF case and the inverse problem resolution must be adapted to the semi-blind deconvolution formulation. The regularization of the subsequent ill-posed problem is achieved using a combination of $\mathcal{L}^{1}$ and $\mathcal{L}^{2}$ terms. On the one hand, the a priori on the reflectivity $\mathrm{x}$ to be restored is enforced via the sparsity constraint $\|\boldsymbol{\Gamma} \mathbf{x}\|_{1}, \boldsymbol{\Gamma}$ being a linear transform. On the other hand, the a priori on the PSF is incorporated as the fidelity-type term $\left\|\mathbf{h}-\mathbf{h}_{\mathbf{0}}\right\|_{2}^{2}$, where $\mathbf{h}_{\mathbf{0}}$ is an initial guess and $\mathbf{h}$ stands for the PSF to be recovered.

Efficient sparsity criteria include, e.g., total variation (TV) [17] for optical and MRI images and Fourier transform [18] for US images. Here, we consider the alternating direction method of multipliers (ADMM) framework, which is a variant of the classical augmented Lagrangian (AL) method 
implementable for optimization problems with separable structures and linear constraints. Recent literature reports efficient methods for general image restoration based on the ADMM approach $[19,20]$. In this paper, we perform the joint estimation of the HR image and the PSF in a semi-blind deconvolution formulation for resolution enhancement. We eventually choose to promote piecewise regular solutions of US images via the edge-preserving TV representation.

\section{NON-BLIND DECONVOLUTION FRAMEWORK AND RELATED WORK}

\subsection{Image Formation Model}

Under the assumption of weak scattering conditions, the classical interaction between the acoustic field and examined tissues is linear [1, 2, 21, 22] and the 2-D ultrasound image formation model is given by

$$
\mathbf{y}=\mathbf{H x}+\mathbf{n}
$$

where $\mathbf{y} \in \mathbb{R}^{n}$ is the vertical concatenation of $n_{1}$ acquired radio frequency (RF) signals of length $n_{2}$ (also known as "lexicographical notation") with $n=n_{1} \times n_{2}, \mathbf{x}_{\mathbf{n}} \in \mathbb{R}^{n}$ denotes the tissue reflectivity function (TRF) to be recovered using the same vertical concatenation as for $\mathbf{y}, \mathbf{H} \in \mathbb{R}^{n \times n}$ stands for the 2-D spatially invariant point spread function (PSF) matrix, and $\mathbf{n} \in \mathbb{R}^{n}$ is the noise. Under circular boundary conditions, $\mathbf{H}$ is a circulant matrix with respect to its first row, i.e., a zero-padded vectorized kernel $\mathbf{h} \in \mathbb{R}^{n}$ of the $\ell=\ell_{1} \times \ell_{2}$ coefficients built from the 2-D PSF, with $\ell_{1} \leq n_{1}$ and $\ell_{2} \leq n_{2}$. The $n^{2}$ coefficients of $\mathbf{H}$ are thus entirely determined by the $\ell$ non-zero elements of $\mathbf{h}$.

Taking into account the low resolution (LR) nature of $\mathbf{y}$ for the so-called SR image restoration [7], we introduce in (1) a matrix $\mathbf{S}$ modelling the loss of information on the observed signal [19]. The image formation model can thus be rewritten as

$$
\mathbf{y}=\mathbf{S H x}+\mathbf{n}
$$

with $\mathbf{x} \in \mathbb{R}^{m}$ the resized HR image, $\mathbf{H} \in \mathbb{R}^{m \times m}$ the PSF matrix with the corresponding zero-padded vectorized kernel $\mathbf{h} \in \mathbb{R}^{m}$, and $\mathbf{S}$ a $n \times m(n \leq m)$ downsampling matrix of range $s_{r}$ in each dimension, containing ones and zeros only [19]. Namely, if $n=n_{1} \times n_{2}$, then $m=s_{r}^{2} \times n=m_{1} \times m_{2}$ with $m_{1}=s_{r} \times n_{1}$ and $m_{2}=s_{r} \times n_{2}$.

\subsection{Inverse Problem Formulation}

When $\mathbf{H}$ is known, the estimation of $\mathbf{x}$ can be formulated in a first attempt as the following minimization problem

$$
\min _{\mathbf{x} \in \mathbb{R}^{m}}\|\mathbf{y}-\mathbf{S H} \mathbf{x}\|_{2}^{2} \text {. }
$$

However, problem (3) is not well-posed since the uniqueness of the solution is not guaranteed. In order to perform its estimation, we need additional information about $\mathrm{x}$ such as, for instance, TV criterion a priori. In this case, the estimation problem within the super-resolution framework becomes

$$
\min _{\mathbf{x} \in \mathbb{R}^{m}}\|\mathbf{\Gamma} \mathbf{x}\| \|_{1} \quad \text { s.t. } \quad\|\mathbf{y}-\mathbf{S H x}\|_{2}^{2} \leq \alpha
$$

or in its equivalent unconstrained form

$$
\min _{\mathbf{x} \in \mathbb{R}^{m}}\|\mathbf{y}-\mathbf{S H} \mathbf{x}\|_{2}^{2}+\tau\|\mid \boldsymbol{\Gamma} \mathbf{x}\| \|_{1} .
$$

$\Gamma$ is a linear transform, e.g., the gradient operator $\nabla$ (TV a priori), and $\tau$ and $\alpha$ are positive real numbers measuring the trade-off between the fit to $\mathbf{y}$ (data fidelity) and the amount of regularization.

Note that since we chose circular boundary conditions, $\mathbf{H}$ is a block-circulant matrix and can hence be diagonalized by Fourier transform (as well as $\boldsymbol{\Gamma}$ for instance in the TV case).

\subsection{Alternating Direction Method of Multipliers}

The solution of (5) can be efficiently computed within the AL framework $[16,19]$. Let us consider the general problem

$$
\begin{array}{cl}
\min & f_{1}(\mathbf{u})+f_{2}(\mathbf{v}) \\
\text { s.t. } & \mathbf{u} \in \mathcal{U}, \mathbf{v} \in \mathcal{V}, \mathbf{A u}+\mathbf{B v}=\mathbf{c}
\end{array}
$$

where $\mathbf{A} \in \mathbb{R}^{p \times q_{1}}$ and $\mathbf{B} \in \mathbb{R}^{p \times q_{2}}$ are given matrices, $\mathbf{c} \in \mathbb{R}^{p}$ is a given vector, $\mathcal{U} \subseteq \mathbb{R}^{q_{1}}$ and $\mathcal{V} \subseteq \mathbb{R}^{q_{2}}$ are given convex sets, $f_{1}: \mathcal{U} \rightarrow \mathbb{R}$ and $f_{2}: \mathcal{V} \rightarrow \mathbb{R}$ are closed convex functions.

Given an initial $\lambda_{0}$, the ADMM method approaches the solution of the original problem (6) by iteratively solving the following problem

$$
\left\{\begin{array}{cccc}
\mathbf{u}_{k+1} & \in \underset{\mathbf{u} \in \mathcal{U}}{\operatorname{argmin}} & \mathcal{L}\left(\mathbf{u}, \mathbf{v}_{k}, \boldsymbol{\lambda}_{k}\right) \\
\mathbf{v}_{k+1} & \in \underset{\mathbf{v} \in \mathcal{V}}{\operatorname{argmin}} & \mathcal{L}\left(\mathbf{u}_{k+1}, \mathbf{v}, \boldsymbol{\lambda}_{k}\right) \\
\boldsymbol{\lambda}_{k+1} & = & \boldsymbol{\lambda}_{k}+\beta\left(\mathbf{A} \mathbf{u}_{k+1}+\mathbf{B} \mathbf{v}_{k+1}-\mathbf{c}\right)
\end{array}\right.
$$

where $\mathcal{L}(\mathbf{u}, \mathbf{v}, \boldsymbol{\lambda})$ denotes the AL function of (6) with $\boldsymbol{\lambda} \in \mathbb{R}^{p}$ the Lagrangian multiplier attached to the linear constraints, and $\beta \in \mathbb{R}_{+}^{*}$ is the penalty parameter for the violation of these constraints. Our problem (4) fits the framework (6) by choosing $\mathbf{u}=\mathbf{x}, \mathbf{v}=\operatorname{vect}([\boldsymbol{\Gamma} \mathbf{x} \mathbf{H} \mathbf{x}]), f_{1}(\mathbf{u})=0, f_{2}(\mathbf{v})=$ $\|\mathbf{\Gamma x} \mid\|_{1}+\chi_{\mathcal{Z}}(\mathbf{H x}), \mathbf{A}=\left[\boldsymbol{\Gamma}^{T} \mathbf{H}^{T}\right]^{T}, \mathbf{B}=-\mathbf{I}_{3 m}$ and $\mathbf{c}=$ $\mathbf{0}_{3 m} . \mathbf{I}_{3 m}$ is the $3 m \times 3 m$ identity matrix, $\mathbf{0}_{3 m}$ stands for a zero vector of length $3 m$, vect $(\cdot)$ denotes the vertical vectorization operation, and $\chi_{\mathcal{Z}}$ is the indicator function of the set $\mathcal{Z}=\left\{\mathbf{z} \in \mathbb{R}^{m}, \mathbf{z}=\mathbf{H x},\|\mathbf{y}-\mathbf{S z}\|_{2}^{2} \leq \alpha\right\}$. Note that in our case, $q_{1}=m$ and $q_{2}=p=3 m$.

\section{PROPOSED SEMI-BLIND DECONVOLUTION IN ULTRASOUND IMAGING}

\subsection{Point Spread Function in Ultrasound Imaging}

Estimation of the US PSF is a fundamental step in both the joint and disjoint estimation approaches. Even slight inaccuracies may cause a severe loss in the restoration performance [6]. In US imaging, a rough estimate of the PSF can 
be directly computed from the RF image based on the minimum phase assumption by inversely mapping a subset of the positive-indexed signal components in the cepstrum signal (i.e., homomorphic filtering) [23, 24]. The PSF can furthermore be considered space-invariant on small enough image segments. Thus, given an initial guess on $\mathbf{h}$ (for instance using the homomorphic filtering method), we propose to overcome the PSF estimation inaccuracies via a robust semi-blind deconvolution framework which consists in performing the estimation of the PSF coefficients jointly with the ones of the HR image.

\subsection{Joint estimation of the PSF and the HR image}

Taking into account both the semi-blind deconvolution and the regularization aspects, we propose to reformulate the estimation problem (5) as follows

$$
\min _{\mathbf{x} \in \mathbb{R}^{m}, \mathbf{h} \in \mathbb{R}^{\ell}}\|\mathbf{y}-\mathbf{S H x}\|_{2}^{2}+\tau\|\mid \boldsymbol{\Gamma} \mathbf{x}\|\left\|_{1}+\gamma\right\| \mathbf{h}-\mathbf{h}_{\mathbf{0}} \|_{2}^{2}
$$

where $\mathbf{h}_{\mathbf{0}}$ is the initial estimate of the PSF and $\gamma, \tau$ are regularization coefficients. Note that a similar $\mathcal{L}^{2}$ fidelity term attached to $\mathbf{h}$ has already been used in a former work related to array calibration [25]. Rather than solving this concurrent minimisation problem, we decompose (8) as a sequential alternating minimization problem with respect to $\mathbf{x}$ and $\mathbf{h}$. It is worth noting that the cost function in (8) is strictly convex with respect to $\mathbf{x}$ or $\mathbf{h}$ but may not be jointly convex with respect to $(\mathbf{x}, \mathbf{h})$. The accuracy of the initial estimates $\mathbf{x}_{\mathbf{0}}$ or $\mathbf{h}_{\mathbf{0}}$ may therefore affect the performance. However, in our experiments, the initialization stage was accurate enough to provide satisfactory results in terms of convergence.

Given an initial $\mathbf{h}_{0}$ (or equivalently $\mathbf{H}_{\mathbf{0}}$ ), our algorithm can then be iteratively computed until convergence as follows

$$
\begin{gathered}
\mathbf{x}_{k+1}=\underset{\mathbf{x} \in \mathbb{R}^{m}}{\operatorname{argmin}}\left\|\mathbf{y}-\mathbf{S H}_{\mathbf{k}} \mathbf{x}\right\|_{2}^{2}+\tau\|\mid \boldsymbol{\Gamma} \mathbf{x}\| \|_{1} \\
\mathbf{h}_{k+1}=\underset{\mathbf{h} \in \mathbb{R}^{\ell}}{\operatorname{argmin}}\left\|\mathbf{y}-\mathbf{S X}_{\mathbf{k}} \mathbf{P h}\right\|_{2}^{2}+\gamma\left\|\mathbf{h}-\mathbf{h}_{\mathbf{0}}\right\|_{2}^{2}
\end{gathered}
$$

where $\mathbf{P} \in \mathbb{R}^{m \times \ell}$ is a simple structure matrix mapping the $\ell$ coefficients of the PSF kernel $\mathbf{h}$ to a $m$ length vector so that $\mathbf{H}_{\mathbf{k}} \mathbf{x}=\mathbf{X}_{\mathbf{k}} \mathbf{P h} . \mathbf{X}_{\mathbf{k}} \in \mathbb{R}^{m \times m}$ is a circulant matrix with the same structure as $\mathbf{H}_{\mathbf{k}}$ where the circulant kernel is $\mathbf{x}_{\mathbf{k}} \in \mathbb{R}^{m}$.

\subsection{Details of Implementation}

On the one hand, at iteration $k+1$, when $\mathbf{h}_{\mathbf{k}}$ is known, the solution of (9a) is given in Section 2.3. A detailed implementation of this stage when $\Gamma=\nabla$ can be found in [16]. It involves pointwise fast Fourier transform (FFT) inversion, soft-thresholding and projection operations which can be efficiently computed. On the other hand, when $\mathbf{x}_{\mathbf{k}}$ is known, Eq. (9b) is a regularized least square problem and its solution can be written as [25]

$$
\mathbf{h}_{k+1}=\left[\left(\mathbf{S X}_{\mathbf{k}} \mathbf{P}\right)^{T}\left(\mathbf{S X}_{\mathbf{k}} \mathbf{P}\right)+\gamma \mathbf{I}\right]^{-1}\left[\left(\mathbf{S X}_{\mathbf{k}} \mathbf{P}\right)^{T} \mathbf{y}+\gamma \mathbf{h}_{\mathbf{0}}\right]
$$

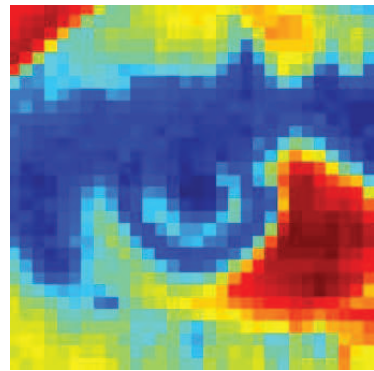

(a) Original HR image

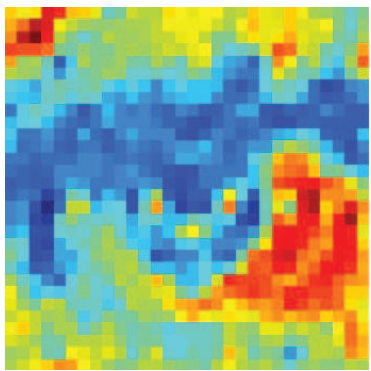

(c) Non-blind formulation

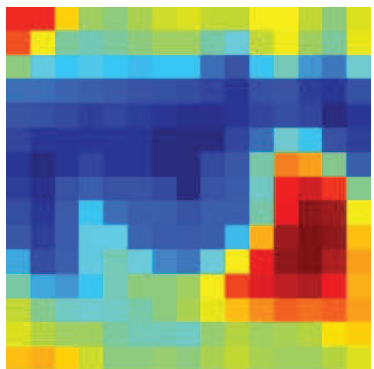

(b) Input LR image

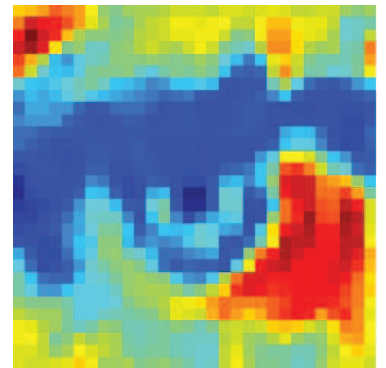

(d) Semi-blind formulation
Fig. 1. Result of semi-blind deconvolution-based resolution enhancement compared with the non-blind case. Input image is a $30 \times 30$ pixel detail of the "Lena" image. Parameters are $s_{r}=2, \alpha=0.01, \beta=0.2$ and $\gamma=4 \times 10^{6}$.

where $\mathbf{I}$ is an identity matrix of size $\ell \times \ell$. Each of the four terms in (10) can be estimated efficiently. $\left(\mathbf{S X}_{\mathbf{k}} \mathbf{P}\right)^{T} \mathbf{y}$ can be computed as the mapping through $\mathbf{P}$ of $\ell$ coefficients of the convolution of the $m$ length vectors $\mathbf{x}$ and $\mathbf{S}^{T} \mathbf{y}$. The matrix $\left(\mathbf{S X}_{\mathbf{k}} \mathbf{P}\right)^{T}\left(\mathbf{S X}_{\mathbf{k}} \mathbf{P}\right)$ can be assembled with $\ell \times \ell$ coefficients properly selected from the $n \times n$ matrix $\left(\mathbf{S X}_{\mathbf{k}}\right)^{T}\left(\mathbf{S X}_{\mathbf{k}}\right)$, which is a cross-correlation matrix of all the $s_{r}$ factor downsampled images, namely, the set $\left\{\mathbf{S x}_{\mathbf{k}}{ }^{i}\right\}, 0 \leq i \leq n-1$, with $\mathbf{x}_{\mathbf{k}}{ }^{i}$ the circular shift of $\mathbf{x}_{\mathbf{k}}$ by $i$ elements.

Note that the first part of (10) is the inversion of a $\ell \times \ell$ matrix $\left(\mathbf{S X}_{\mathbf{k}} \mathbf{P}\right)^{T}\left(\mathbf{S X}_{\mathbf{k}} \mathbf{P}\right)$ regularized by $\gamma \mathbf{I}$, where $\gamma$ is a parameter involved in both a diagonal loading process and a fit to $\mathbf{h}_{\mathbf{0}}$. High values of $\gamma$ will result in an estimation of $\mathbf{h}$ close to the initial PSF $\mathbf{h}_{\mathbf{0}}$ while small values of $\gamma$ will let the estimation mainly rely on the data fidelity term.

\section{EXPERIMENTS, RESULTS AND DISCUSSION}

\subsection{Optical Images}

To get a better insight into the algorithm performance, we first address the restoration of an optical synthetic image where the degradation process (e.g., the additive noise and the PSF) is known. Fig. 1 gives an example of such an experiment, where a $30 \times 30$ pixel block of the "Lena" image was processed in the known PSF (with the true $\mathbf{h}$ ), non-blind viz initial guess (with $\mathbf{h}_{\mathbf{0}}$ ), and semi-blind (with $\mathbf{h}_{\mathbf{k}}$ when convergence is reached) 

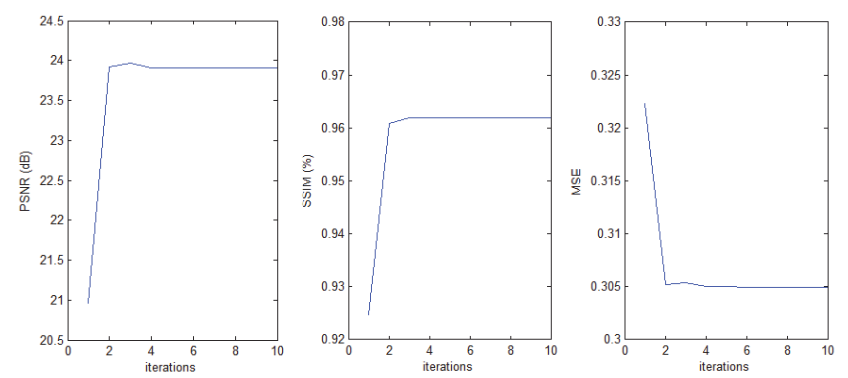

Fig. 2. Algorithm performance addressed in terms of (left) PSNR, (middle) SSIM, and (right) PSF mean-square error. 10 iterations of (9) are often enough.

deconvolution cases. Note that the true PSF $\mathbf{h}$ and its initial guess $\mathbf{h}_{\mathbf{0}}$ have both a Gaussian shape but different standard deviations (respectively $\sigma=1.25$ and $\sigma_{0}=3.75$ ). As can be seen, the fine details of the eye structure are better recovered in the semi-blind formulation. Though not depicted here due to page limitation, it was observed that the final estimate $\mathbf{h}_{\mathbf{k}}$ is much closer to the true PSF than $\mathbf{h}_{\mathbf{0}}$ in terms of visual inspection and mean-square error (MSE).

Performance and convergence of the algorithm (9) is evaluated in Fig. 2 using classical criteria such as peak signal-tonoise ratio (PSNR), structural similarity (SSIM) [26] and relative PSF mean-square error. Compared with the non-blind case (i.e., when $k=0$ ), significant improvements are observable with respect to all criteria. Computation time for the semi-blind deconvolution (9) of this image is approximately 2 seconds with a Matlab implementation and a $2.4 \mathrm{GHz}$ desktop computer.

\subsection{Synthetic Ultrasound Data}

We investigate in this section the processing of simple US synthetic data simulated with a realistic PSF. A synthetic US signal was generated with the FIELD II software [21]. The true PSF $\mathbf{h}$ has a central frequency of $f=3 \mathrm{MHz}$ whereas the input PSF $\mathbf{h}_{\mathbf{0}}$ central frequency was set to $f_{0}=3.1 \mathrm{MHz}$ in order to model the PSF estimation error. The sampling frequency is set to $f_{s}=30 \mathrm{MHz}$. The signal to be restored consists of 5 single scatterers, shown by the vertical lines in Fig. 3.

Computation time of the proposed method is about $1 \mathrm{sec}-$ ond. Note that in the non-blind case, the deconvolution of US data with an inaccurate PSF provides results often worse than the raw input data. Our semi-blind deconvolution technique (9) for resolution enhancement always provides results better than the non-blind deconvolution approach, as can be seen in Fig. 3. The axial resolution enhancement of our method is evaluated in Table 1 in terms of full width at half maximum (FWHM) and resolution gain (RG) defined in [11]. RG was defined by the ratio between the number of samples of the normalized autocorrelation function with values higher than
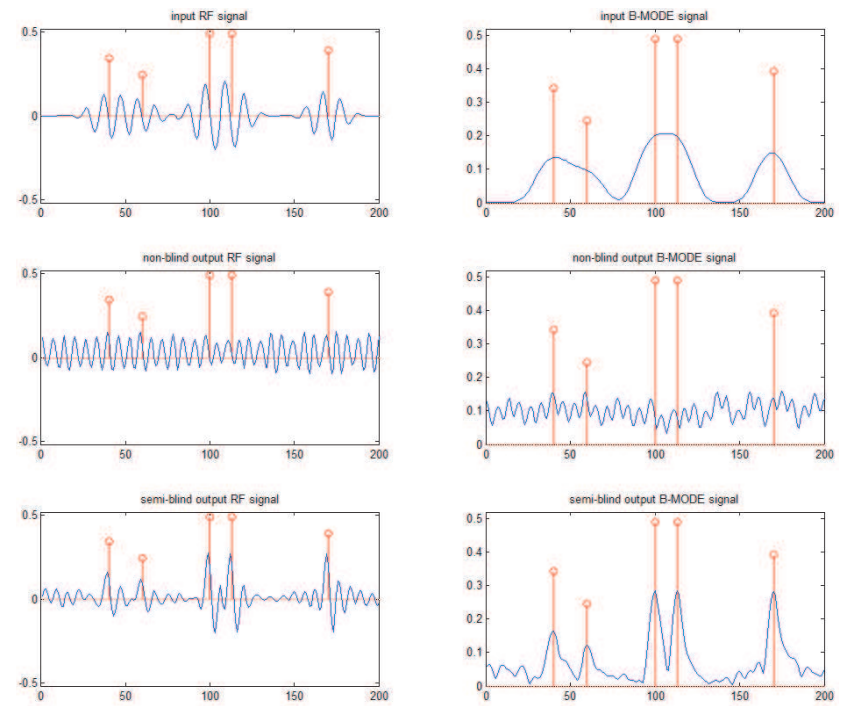

Fig. 3. Resolution of US scatterers (vertical lines) with a simulated RF signal. Figure shows (top row) the input signal, (middle row) the non-blind and (bottom row) the semi-blind deconvolutions. Results are displayed in RF (left column) and B-mode (right column) representations. Parameters are $s_{r}=1, \alpha=0.5, \beta=0.1$ and $\gamma=2 \times 10^{2}$.

Table 1. Performance of our method in terms of full width at half maximum (FWHM) and resolution gain (RG) [11].

\begin{tabular}{cccc}
\hline & $\begin{array}{c}\text { Input } \\
\text { image }\end{array}$ & $\begin{array}{c}\text { Non-blind } \\
\text { deconvolution }\end{array}$ & $\begin{array}{c}\text { Semi-blind } \\
\text { deconvolution }\end{array}$ \\
\hline FWHM & 24 & 10 & 8 \\
RG & 1.00 & 0.34 & 2.78 \\
\hline
\end{tabular}

0.75, computed for the envelope input signal, and that for the deconvolved output signal.

Both criteria show significant improvements compared with the non-blind deconvolution approach. Furthermore, the two scatterers in the middle part of the original signal are recovered, demonstrating an improved spatial resolution.

\section{CONCLUSION}

In this paper, we have proposed an algorithmic framework for semi-blind deconvolution-based resolution enhancement. The technique consists in the joint estimation of the original image and blur. Prior knowledge about the PSF and the image is incorporated via regularization techniques. Interestingly, the algorithm can be implemented in a computationally efficient manner. Performance and robustness have been currently assessed on optical images and synthetic US data through qualitative and quantitative criteria. It was shown that our approach leads to resolution improvement and provides the recovery of close scatterers in the case of simple US data. The results are promising and will be extended to more realistic US images and to real in vivo data in a future work. 


\section{REFERENCES}

[1] T. L. Szabo, Diagnostic ultrasound imaging: inside out, Biomedical Engineering Series. Elsevier Academic Press, 2004.

[2] J. L. Prince and J. M. Links, Medical imaging signals and systems, Pearson Prentice Hall, 2006.

[3] G. T. Clement, J. Huttunen, and K. Hynynen, "Superresolution ultrasound imaging using back-projected reconstruction," $J$. Acoust. Soc. Am., vol. 118, no. 6, pp. 3953 - 3960, Sept. 2005.

[4] M. A. Ellis, F. Viola, and W. F. Walker, "Super-resolution image reconstruction using diffuse source models," Ultrasound in Med. \& Biol., vol. 36, no. 6, pp. 967 - 977, 2010.

[5] T.F. Chan and Chiu-Kwong Wong, "Total variation blind deconvolution," IEEE Trans. Image Process., vol. 7, no. 3, pp. 370 - 375, March 1998.

[6] T. Taxt, "Restoration of medical ultrasound images using twodimensional homomorphic deconvolution," IEEE Trans. Ultrason. Ferroelectr. Freq. Control, vol. 42, no. 4, pp. 543 - 554, July 1995.

[7] S. C. Park, M. K. Park, and M. G. Kang, "Super-resolution image reconstruction: a technical overview," IEEE Signal Process. Mag., vol. 20, no. 3, pp. 21 - 36, May 2003.

[8] R. Morin, A. Basarab, M. Ploquin, and D. Kouamé, "Post-processing multiple-frame super-resolution in ultrasound imaging," in SPIE Medical Imaging, 2012.

[9] L. Wang, L. Xiao, J. Zhang, and Z. Wei, "New image restoration method associated with tetrolets shrinkage and weighted anisotropic total variation," Signal Processing, vol. 93, no. 4, pp. $661-670,2013$.

[10] H. Ji and K. Wang, "Robust image deblurring with an inaccurate blur kernel," IEEE Trans. Image Process., vol. 21, no. 4, pp. 1624 - 1634, Apr. 2012.

[11] O. Michailovich and A. Tannenbaum, "Blind deconvolution of medical ultrasound images: A parametric inverse filtering approach," IEEE Trans. Image Process., vol. 16, no. 12, pp. 3005 - 3019, Dec. 2007.

[12] S. Dolui and O. V. Michailovich, "Blind deconvolution of medical ultrasound images using variable splitting and proximal point methods," in Proc. IEEE Int. Symp. Biomedical Imaging: From Nano to Macro, Apr. 2011, pp. 1 - 5.

[13] C. Yu, C. Zhang, and L. Xie, "A blind deconvolution approach to ultrasound imaging," IEEE Trans. Ultrason. Ferroelectr. Freq. Control, vol. 59, no. 2, pp. 271 - 280, Feb. 2012.

[14] D. Kundur and D. Hatzinakos, "Blind image deconvolution," IEEE Signal Process. Mag., vol. 13, no. 3, pp. 43 - 64, May 1996.

[15] A.K. Takahata, E.Z. Nadalin, R. Ferrari, L.T. Duarte, R. Suyama, R.R. Lopes, J.M.T. Romano, and M. Tygel, "Unsupervised processing of geophysical signals: A review of some key aspects of blind deconvolution and blind source separation," IEEE Signal Process. Mag., vol. 29, no. 4, pp. 27 - 35, July 2012.
[16] R. Morin, A. Basarab, and D. Kouamé, "Alternating direction method of multipliers framework for super-resolution in ultrasound imaging," in Proc. IEEE Int. Symp. Biomedical Imaging: From Nano to Macro, 2012, pp. 1595 - 1598.

[17] L. I. Rudin, S. Osher, and E. Fatemi, "Nonlinear total variation based noise removal algorithms," Physica D: Nonlinear Phenomena, vol. 60, pp. 259-268, 1992.

[18] C. Quinsac, A. Basarab, and D. Kouamé, "Frequency domain compressive sampling for ultrasound imaging," Advances in Acoustics and Vibration, vol. 12, pp. 1 - 16, 2012.

[19] M. Ng, P. Weiss, and X.-M. Yuang, "Solving constrained totalvariation image restoration and reconstruction problems via alternating direction methods," SIAM journal on Scientific Computing, vol. 32, 2010.

[20] M. V. Afonso, J. M. Bioucas-Dias, and M. A. T. Figueiredo, "An augmented Lagrangian approach to the constrained optimization formulation of imaging inverse problems," IEEE Trans. Image Process., vol. 20, no. 3, pp. 681 - 695, March 2011.

[21] J. A. Jensen, "A model for the propagation and scattering of ultrasound in tissue," J. Acoust. Soc. Am., vol. 89, no. 1, pp. 182 - 191, Jan. 1991.

[22] J. A. Jensen, J. Mathorne, T. Gravesen, and B. Stage, "Deconvolution of in-vivo ultrasound B-mode images," Ultrason. Imaging, vol. 15, pp. 122 - 133, 1993.

[23] J. A. Jensen and S. Leeman, "Nonparametric estimation of ultrasound pulses," IEEE Trans. Biomedical Engineering, vol. 41, no. 10, pp. 929 - 936, Oct. 1994.

[24] A. V. Oppenheim and R. W. Schafer, Discrete-time signal processing, Upper Saddle River (N.J.), Prentice Hall, 1989.

[25] O. Besson, S. Bidon, and C. L. Tournemine, "Robust approaches to remote calibration of a transmitting array," in Signal Processing, 2010, vol. 90, pp. 1373 - 1381.

[26] Z. Wang, A. C. Bovik, H. R. Sheikh, and E. P. Simoncelli, "Image quality assessment: from error visibility to structural similarity," IEEE Trans. Image Process., vol. 13, no. 4, pp. $600-612$, April 2004. 